\title{
Beam Energy Evolution of HBT Systematics at the AGS
}

\author{
M.A. Lisa ${ }^{+}$, for the E895 Collaboration* \\ +Physics Department, Ohio State University, 174 W. 18th Ave., Columbus, OH 43210
}

We present preliminary results of the first $\pi$ interferometry (HBT) excitation function at intermediate AGS energies. The beam energy evolution of the correlations' dependence on $m_{T}$, centrality, and emission angle with respect to the reaction plane are discussed. Comparisons with predictions of the RQMD cascade model are made.

Two-particle intensity interferometry (HBT) measurements have long been used to study the geometry and dynamics of heavy ion collisions (see, e.g. [1]). Pion correlation functions are sensitive to the pion source size, shape, decay-time, and long-lived particle (e.g. $\Lambda$ ) production. In addition, dynamic effects such as flow produce space-momentum correlations resulting in dependences of the correlation functions on $\pi$ momentum.

In this paper, we discuss an excitation function $(2-8 \mathrm{AGeV})$ of $\pi^{-}$HBT measurements. Studying the evolution of the correlations as a function of $E_{\text {beam }}$ is important for two reasons. Firstly, a sudden increase, at some $E_{\text {beam }}$, in the lifetime of the hadronic fireball has long been proposed as a robust signal of the onset of QGP formation [2 [4]. Secondly, the sensitivity of correlation functions to the underlying physics makes such measurements potent tools to test the dynamics of microscopic models of heavy ion collisions. Many models attempt to extrapolate to the RHIC energies. Confidence in the ability to extrapolate (determined by the correct underlying physics and its evolution with energy) would be enhanced if the model reproduces an excitation function of detailed HBT systematics.

Using the large-acceptance EOS Time Projection Chamber [5] the E895 collaboration measured charged particles from $\mathrm{Au}+\mathrm{Au}$ collisions at 2, 4, 6, and $8 \mathrm{AGeV}$ at the Brookhaven AGS. Good particle identification minimized $e^{-}$contamination of the $\pi^{-}$ sample. Momentum resolution, largely due to multiple Coulomb scattering and straggling in the $3 \%$ interaction length target, was on the order of $1.5-3 \%$. The experimental correlation functions have been corrected for the momentum resolution with an iterative method similar to that employed by the NA44 collaboration [6]. This correction typically increases the fitted $\lambda$ parameter by $15 \%$, and the radii by $5 \%$.

Track merging and splitting effects were eliminated by a two-track geometrical cut based on the tracking algorithm. As expected, this cut discards some pairs (in the "real" and event-mixed distributions) at low relative momentum, q. However, due to detector geometry, this cut preferentially discards low-q pairs at high $p_{T}$; thus, to minimize phasespace bias effects, we restrict our analysis to low $p_{T}$ and use narrow windows in $p_{T}$.

A full Coulomb-wave integration [7,8] over a spatial source of 5-fm Gaussian radius was used to generate the Coulomb correction, which was applied pair-wise to the event-mixed

\footnotetext{
*Author list and Grant acknowledgements may be found in G. Rai's contribution to these Proceedings.
} 
denominator of the correlation function. Identical Coulomb correction functions were applied to data and to correlation functions from the RQMD (see below). Further details of the analysis have been reported previously [9].

The high quality of the data is seen in Figure 1, where projections in the Bertsch-Pratt (BP), or out-side-long, system are shown for midrapidity pions from central events at each bombarding energy. The relative momentum $q$ was calculated in the fixed $\mathrm{Au}+\mathrm{Au}$ c.m. frame. The functional form

$$
C\left(q_{\text {out }}, q_{\text {side }}, q_{\text {long }}\right)=1+\lambda e^{-R_{\text {out }}^{2} q_{\text {out }}^{2}-R_{\text {side }}^{2} q_{\text {side }}^{2}-R_{\text {long }}^{2} q_{\text {long }}^{2}-2 R_{\text {ol }}^{2} q_{\text {out }} q_{\text {long }}}
$$

was fit to the data, using a maximum-likelihood technique [10]. The cross-term [11] $R_{o l}^{2}$ was consistent with zero in all cases and uncorrelated with the other parameters.

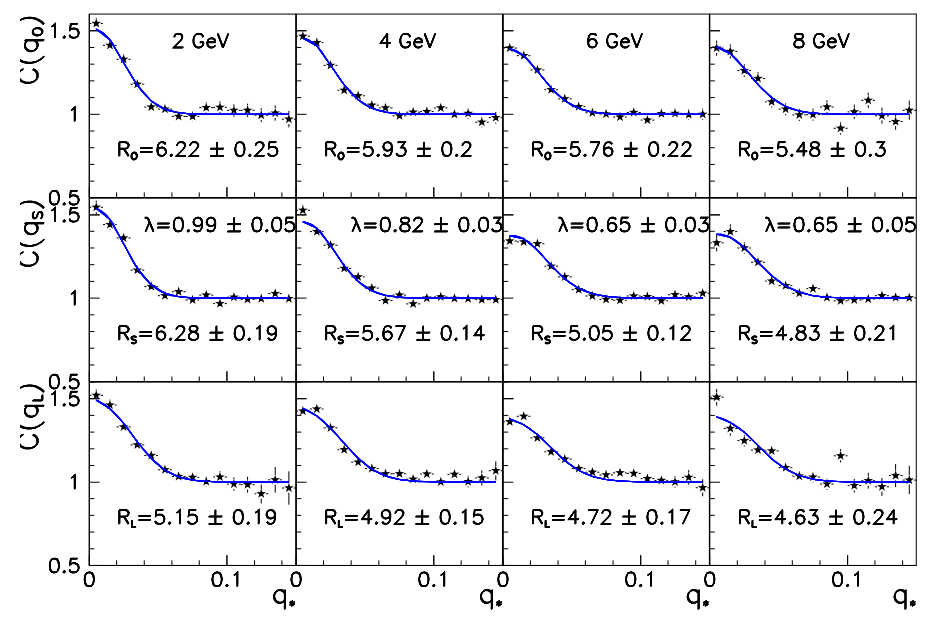

Figure 1. Projections of the 3-D $\pi^{-}$correlation functions from central collisions $\left(\sim 11 \% \sigma_{T}\right)$. $q_{\text {out }}$ (top), $q_{\text {side }}$ (middle), and $q_{\text {long }}$ (bottom) projections are shown for collisions at all beam energies measured. $y=y_{c m} \pm 0.35$ and $p_{T}=0.1-0.3 \mathrm{GeV} / \mathrm{c}$ cuts were applied. Projections in a given q-component are integrated over $\pm 30 \mathrm{MeV} / \mathrm{c}$ in the other components. Superimposed curves show projections of 3-D fits described in text.

Correlation functions were also constructed in the Yano-Koonin-Podgoretskii (YKP) decomposition [12]; here, the effective lifetime $R^{0}$ is fit more directly. We fit to the form

$$
C\left(q_{0}, q_{\perp}, q_{\|}\right)=1+\lambda e^{-R_{\perp}^{2} q_{\perp}^{2}-R_{\|}^{2}\left(q_{\|}^{2}-q_{0}^{2}\right)-\left(R_{o}^{2}+R_{\|}^{2}\right)(q \cdot U)^{2}}
$$

The excitation function of the fit results is presented in Figure 2. Also shown are results of fits to correlation functions generated by using the $\pi^{-}$freeze-out points from the RQMD (v2.3) model [13] as input to the two-particle correlator code CRAB [14]. Both the data and the model show a decrease in the $\lambda$ parameter, due to increased production of long-lived $\pi^{-}$-emitting particles at higher energy [7].

While the longitudinal radii, $R_{\text {long }}$ and $R_{\|}$, display little dependence on beam energy, the observed decrease of the transverse radii $R_{\text {side }}$ and $R_{\text {out }}$ comes as something of a surprise. At low energy, the YKP fits suggest that the model produces a $\pi^{-}$source that is too small and too long-lived; this leads in the BP decomposition to an underprediction in $R_{\text {side }}$, but a reasonable agreement in $R_{\text {out }}$, as the space and time effects partially cancel. 


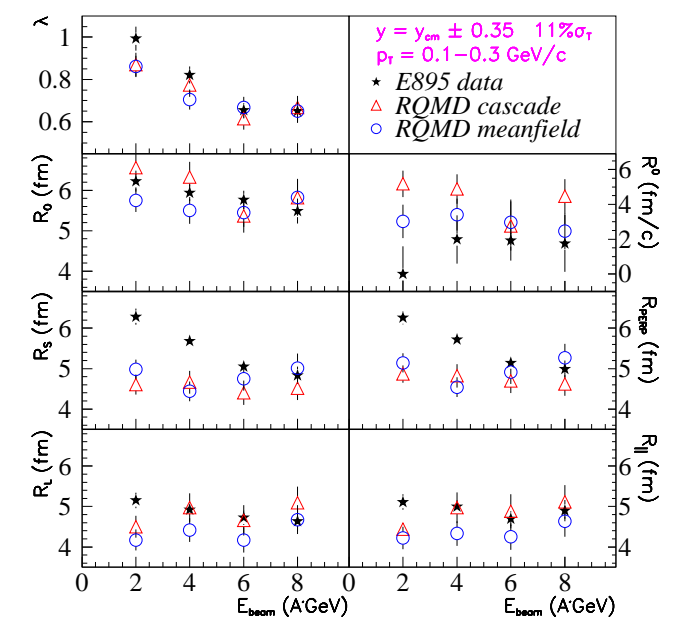

Figure 2. Beam energy dependence of the B-P and YKP fit parameters.

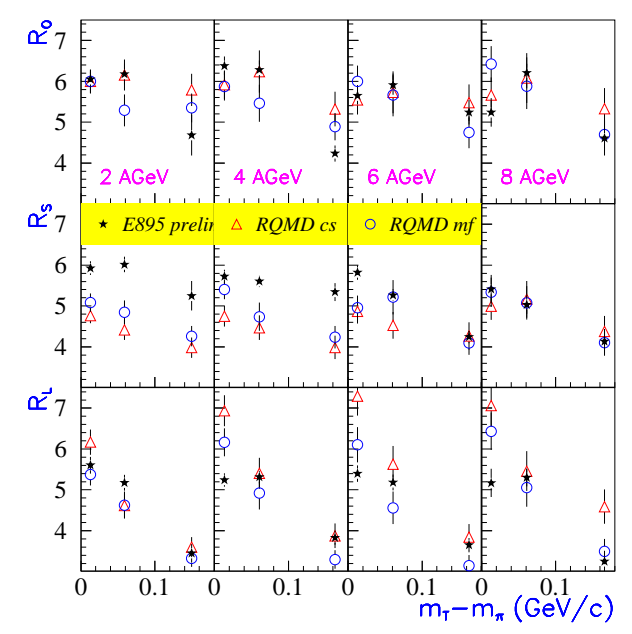

Figure 3. $m_{T}$ dependence of the B-P radii for data and $R Q M D$.

Part of the reason for the decrease in the apparent transverse size is revealed in Figure 3 . It is clear that the $m_{T}$-dependence of $R_{\text {side }}$ (and the space-momentum correlation that causes it) becomes stronger with $E_{\text {beam }}$ in the data, while the model suggests a $m_{T^{-}}$ dependence rather independent of energy, and reminiscent of trends at higher energy. The observed trend may suggest that collective transverse flow builds with bombarding energy, and is only strong enough to affect $R_{\text {side }}\left(m_{T}\right)$ above $E_{\text {beam }} \sim 4 \mathrm{AGeV}$.

Although dynamics determines the $m_{T}$-dependence of the radii, it is worthwhile to check that the HBT radii track somewhat with geometry. Figure 1 shows the impact parameter $(b)$ dependence of the HBT radii ( $b$ was estimated from the charged particle multiplicity). $\lambda$ increases with $b$, as the production of long-lived $\pi$-emitting particles is suppressed relative to direct pions. The transverse radii $\left(R_{\text {side }}\right.$ and $\left.R_{\text {out }}\right)$ decrease for more peripheral collisions, as expected, while $R_{\text {long }}$ shows little $b$-dependence. The trends suggest that the measured pion source reflects the overlap volume of the colliding nuclei.

More detailed information may be obtained by studying the HBT signal as a function of $\pi^{-}$emission angle with respect to the reaction plane. The reaction plane is calculated only from momenta of $\mathrm{Z} \leq 2$ nuclei, for every event [15], so auto-correlations are not an issue. From an overlap-volume picture, one expects an anisotropic apparent shape in the transverse direction for non-central collisions, with a larger spatial scale perpendicular to the reaction plane. Deviations may reflect the non-isotropic flow dynamics of the system prior to freeze-out, or may carry information concerning the opacity of the source [16.

Preliminary results for 2,4 , and $6 \mathrm{AGeV}$ collisions at $b \approx 5-7 \mathrm{fm}$ are shown in Fig. 5. At the lower energy, $R_{\text {side }}$ (the radius most closely related to geometry [2]) exhibits a $\phi_{r p^{-}}$ dependence consistent with geometric considerations. While RQMD simulations (with perfect reaction plane resolution) display similar trends at all energies, at higher energy, the $R_{\text {side }}$ oscillation is not seen in our data. Since the radii are not corrected for the finite dispersion, this is due at least in part to the worsening resolution with which the reaction plane is measured. Further study of this novel HBT signal is required.

In summary, we are mapping out the systematics of pion correlations in the energy 


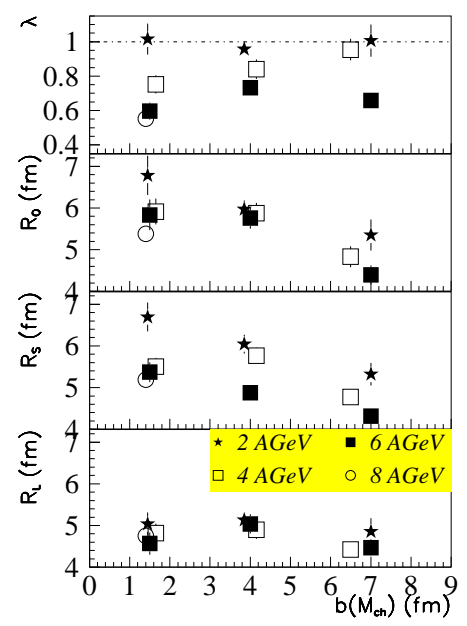

Figure 4. Centrality dependence of the BP fit parameters.

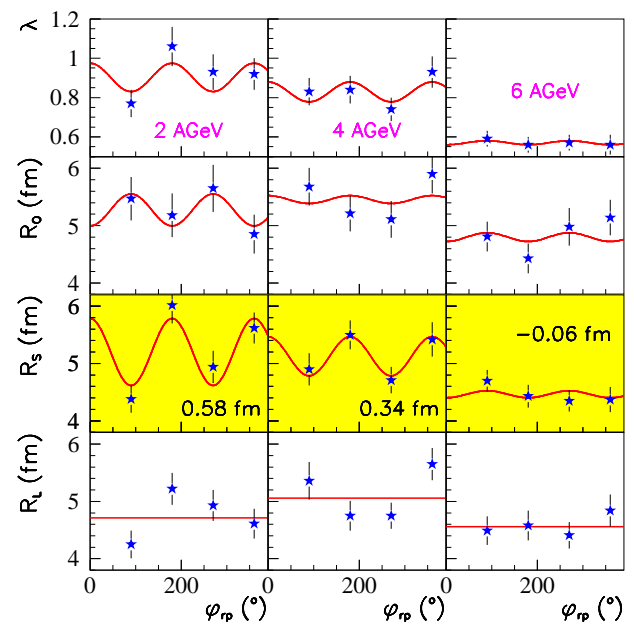

Figure 5. BP parameters versus $\pi^{-}$emission angle with respect to reaction plane.

range between the Bevalac and maximum AGS energy. Large jumps in source size or lifetime at some collision energy, which might indicate the onset of QGP formation, are not observed. Surprisingly, the apparent source size is larger at the lower beam energies; this appears largely a consequence of weaker space-momentum correlations there. The RQMD model, with or without meanfield effects, does not reproduce the data; in the model at low energy, the effective size is too small, the lifetime too large, and $R\left(m_{T}\right)$ does not evolve with beam energy. The impact parameter dependence of the radii follows naive expectations from geometry. An HBT analysis correlated with the event-wise reaction plane reveals a significant oscillation in $R_{\text {side }}$, at low beam energy.

\section{REFERENCES}

1. W. Bauer, C.K. Gelbke, and S. Pratt, Ann. Rev. Nucl. Part. Sci. 42, 77 (1992).

2. S. Pratt, Phys. Rev. C49 2772 (1994), Phys. Rev. D33 1314 (1986)

3. G. Bertsch, M. Gong, and M. Tohyama, Phys. Rev. C37 1896 (1988)

4. D.H. Rischke, Nucl. Phys. A610, 88c (1996)

5. H. Pugh, et al., LBL-22314 (1986); G. Rai, et al., IEEE Trans. Nucl.Sci. 37, 56 (1990)

6. H. Bøggild, et al. (NA44), Phys. Lett. B302, 510 (1993).

7. S. Pratt, T. Csörgő, and J. Zimányi, Phys. Rev. C42, 2646 (1990)

8. A. Messiah, Quantum Mechanics, North-Holland (1961)

9. M.A. Lisa, et al. (E895), proceedings of 15th Winter Workshop on Nuclear Dynamics, Park City, UT, W. Bauer and G. Westfall, eds., Kluwer Academic Press (1999).

10. J. Barrette, et al. (E877), Nucl. Phys A610, 227c (1996)

11. S. Chapman, P. Scotto, and U. Heinz, Phys. Rev. Lett. 22, 4400 (1995).

12. U. Heinz, Nucl. Phys. A610, 264c (1996).

13. H. Sorge, Phys. Rev. C52, 3291 (1995)

14. S. Pratt, Nucl. Phys. A566, 103c (1993).

15. C. Pinkenburg et al. (E895), to appear in Phys. Rev. Lett. 83 (1999)

16. H. Heiselberg, Phys. Rev. Lett. 82, 2052 (1999) 